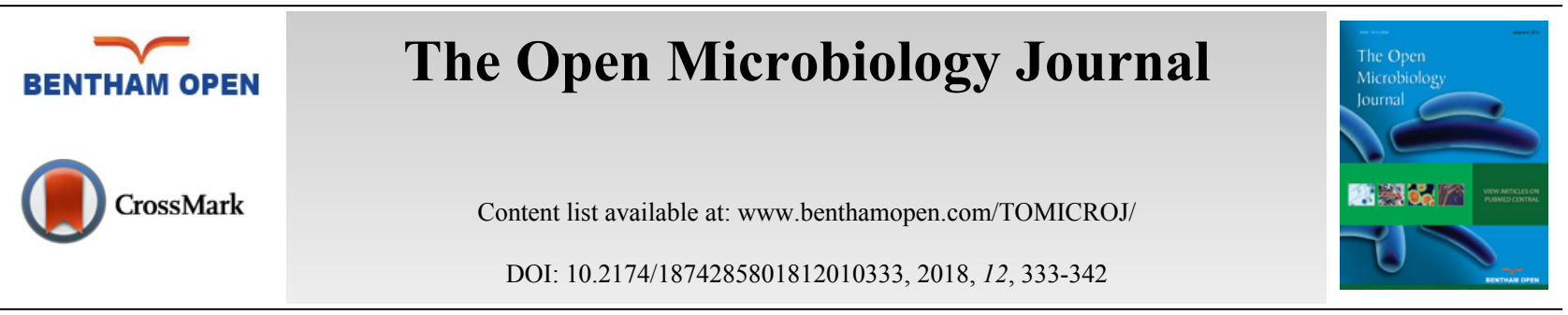

RESEARCH ARTICLE

\title{
Pulmonary Tuberculosis and Associated Factors Among Diabetic Patients Attending Hawassa Adare Hospital, Southern Ethiopia
}

\author{
Ademe Abera ${ }^{1}$ and Gemechu Ameya ${ }^{2, *}$ \\ ${ }^{1}$ Hawassa Comprehensive Specialized Hospital, College of Medicine and Health Sciences, Hawassa University, \\ Hawassa, Ethiopia \\ ${ }^{2}$ Department of Medical Laboratory Science, College of Medicine and Health Sciences, Arba Minch University, Arba \\ Minch, Ethiopia
}

Received: June 18, 2018

Revised: September 20, 2018

Accepted: September 23, 2018

\begin{abstract}
:
Background:

Developing countries have a high burden of Tuberculosis (TB); although it is considered as a disease of the past in most developed countries. The end TB strategy was predicted to stabilize or drop the incidence of TB. However, the rising of the prevalence of immune-related diseases like Diabetes Mellitus (DM) are challenging the TB control strategy in high TB burden region. The objective of this study was to determine the prevalence and associated factors of pulmonary tuberculosis in DM patients attending Adare Hospital, south Ethiopia.
\end{abstract}

\section{Methods:}

A cross-sectional study was carried out on 207 randomly selected diabetic patients at the Adare hospital. A structured pre-tested questionnaire was used during the data collection from participants. Sputum concentration technique followed by Ziehl-Neelsen staining method was used to examine pulmonary tuberculosis. Logistic regression analysis was used to assess the association between various variables and pulmonary tuberculosis. Odds ratios and 95\% CI were computed to determine the strength and presence of the association.

\section{Results:}

The prevalence of pulmonary tuberculosis among diabetics was 5.3\% [95\% CI: $(2.2,8.4)]$. Diabetic patients who were underweight $[\mathrm{AOR}=9.94,95 \% \mathrm{CI}:(1.51-80.89)]$, had more than 10 years duration with DM $[\mathrm{AOR}=7.03$ 95\% CI: (1.357, 73.6)], Alcohol drinking habit $[\mathrm{AOR}=12.49,95 \% \mathrm{CI}:(3.28,77.94)]$, and history of contact with TB [AOR $=5.35,95 \%$ CI: $(1.1-39.12)]$ were the factors positively associated with pulmonary TB infection while being HIV seronegative had a negative association with pulmonary TB infection $[\mathrm{AOR}=0.074,95 \% \mathrm{CI}:(0.001-0.29)]$.

\section{Conclusion:}

High proportion of pulmonary TB was observed in diabetic patients as compared to the national estimated prevalence of TB in the total population. Duration of patient with DM, being underweight, alcohol consumption habit, and contact history with TB were positively associated with pulmonary TB infection while being HIV seronegative had a negative association with the infection in diabetic patients.

Keywords: Associated factors, Diabetic mellitus, Pulmonary tuberculosis, Ethiopia, HIV seronegative.

\footnotetext{
"Address correspondence to this author at the Department of Medical Laboratory Science, College of Medicine and Health Sciences, Arba Minch University, Arba Minch, Ethiopia; Tel: +251-91-783-7681; E-mail: gemechuameya@gmail.com
} 


\section{BACKGROUND}

Tuberculosis is the major public health problem. Different efforts are undertaken to control TB infection, but it is still the major cause of mortality and morbidity in developing courties like Ethiopia. In 2014 global report, there were 9.6 million people who developed active tuberculosis and 1.5 million death cases were reported. Ethiopia which was one of the high burden TB countries met the 2015 WHO target for incidence, prevalence and mortality, even though Africa has still the high burden of TB per total population. According to the World Health Organization (WHO) 2015 report, Africa was the most affected continent by TB which accounts 281 per 100, 000 people while global average TB case report was 133 per 100,000 people. Worldwide, about $20 \%$ of previously treated TB cases and $3.3 \%$ of new cases have multi-drug resistance TB according to the WHO report [1].

Only ten percent of Mycobacterium tuberculosis-infected people will develop active pulmonary disease in their lifetime. Diabetics and people suffering from other chronic diseases have a much higher incidence of disease than the general population [2]. Diabetes Mellitus is a chronic metabolic disease which occurs when the human body does not respond to the insulin that is produced insulin or when the body is not able to produce enough insulin. Worldwide, people suffering from diabetes are estimated to be 422 million with the prevalence of $8.5 \%$ among over 18 years of age according to $2014 \mathrm{WHO}$ report. Its prevalence has been rising more rapidly in developing countries [3]. There were over 1.33 million cases of diabetesc cases in Ethiopia according to the 2015 international diabetic federation report [4].

The association between Diabetes Mellitus (DM) and tuberculosis has been documented long time ago. Their association can be the next challenge for global tuberculosis control. Enhanced understanding of the bidirectional relationship of diabetics and tuberculosis is necessary for proper planning and collaboration to reduce the dual burden of the diseases. Developing countries are still combating with tuberculosis and DM [5]. The incidence rate of TB is very high in sub-Saharan Africa in which Ethiopia islocated. According to 2015 WHO global TB report, prevalence and incidence of TB cases were estimated to be 200 and 207 per 100000 people, respectively [1].

Diabetes mellitus impairs immune function that specifically affects cell mediated immunity, has been associated with low levels of polymorphoneutrophils and reduce the cytokine level of T-helper1 response to TB. In diabetes, macrophage function is inhibited, phagocytic and chemotactic abilities are impaired despite the immune responses being important for tuberculosis inhibition. Therefore, compromised immunity due to diabetes mellitus would conceivably gives an opportunity for TB development [6]. In the coming decades, the end TB strategy predicted to stabilize drop incidence of TB in most parts of the world. Therefore, the rising burden of diabetes has a relative contribution to the high prevalence of TB in developing countries [7 - 9].

Diabetes Mellitus increase the risk .developing latent and active tuberculosis infection, and it complicates the treatment of active TB [8]. It is difficult to treat TB infection in the presence of poor glucose control. On the other hand, delayed sputum culture conversion affects the diagnosis, it also increases the case fatality rate of the patient on treatment and relapse rate after the successful completion of treatment. On the other hand, a study showed that TB treatment reduced the efficiency and concentration of diabetes medications, which results in complicatedness in disease control $[8,10,11]$. In Ethiopia now a day, most health centers provide Acid-Fast Bacilli (AFB) stain of sputum smear for pulmonary TB diagnosis, whilst culture is carried out in limited laboratories [12].

In a previous study, prevalence and factor associated with TB infection among DM patients have been documented [13 - 16]. However, little is known about the epidemiology of pulmonary TB among diabetic people in Ethiopia. There is no study conducted on pulmonary TB among diabetic patients in southern Ethiopia where this study was conducted. Therefore, the aim of this study is to determine the magnitude and associated factors of pulmonary tuberculosis in diabetic patients attending Adare Hospital, southern Ethiopia.

\section{METHODS}

\subsection{Study Settings and Design}

A cross-sectional study was conducted to assess the prevalence of pulmonary tuberculosis and associated factors in diabetic patients attending Hawassa Adare hospital, southern Ethiopia. Hawassa is located $275 \mathrm{~km}$ to the south of Addis Ababa. It is the capital city of the Southern Nations, Nationalities, and Peoples Regional state. According to the 2007 census report, the region has a total population of 15,321,000 [17]. The hospital is giving clinical service for more than half a million people in its districts. There were 800 diabetic patients who monitor their disease status in the hospital. Diabetic patients with clinical indications of TB are examined and those found infected are also managed in the TB and 
leprosy clinic in the hospital. The study period was from March to May 2015.

\subsection{Study Population}

Diabetic patients who were on follow-up in Adare hospital and who had the registration were included in the study. Then people with full information and on follow-up for DM who were above 15 years old were included. However, those who attained diabetic clinic for less than a month, critically ill patient and kyphoscoliosis (difficult to measure height) patient were excluded from the study.

\subsection{Sample Size and Sampling Procedures}

The sample size was calculated using single population proportion formula: $n=(\underline{Z} \alpha / 2)^{2} * p(1-p) / w^{2}$, by considering the following assumptions: the proportion $(\mathrm{p}=0.062)$ was taken from the previous study [18 - 20] of prevalence of TB among diabetic patients in the previous study which was $6.2 \%, 3 \%$ marginal error and $10 \%$ non-response rate was taken. Therefore, the total number of the sample size was 207.

Systematic random sampling technique was employed to sample the patients using unique diabetic follow-up number. The subject participated in the study was selected by $\mathrm{K}^{\text {th }}$ number of patients. There were about 800 diabetic patients attending the hospital. Thus: $\mathrm{K}=800 / 207 \approx 4$. Therefore, the study participant was selected in every 4 patients according to the diabetic's unique numbers of their registration book.

\subsection{Data Collection Tools and Procedures}

Data collection tool was developed after reviewing the literature and all the potential variables related to the objective of the study were included $[5,6,8,13-16,21-32]$. The data collection tool was tested on $5 \%$ of similar study participants before being used in another health facility which had a diabetic clinic to modify the instrument. The questionnaire was designed to obtain information related to the socio-demographic characteristics of study participants, behavioral, nutritional, and medical characteristic. Nutritional status of patients on diabetic follow up was assessed by measuring weight and height of the participants.

\subsection{Specimen Collection and Laboratory Investigations}

Diagnosis of pulmonary tuberculosis among diabetic patients was done according to the national TB diagnosis guideline [19]. All participants provided three consecutive sputum samples (spot-morning-spot) using dry, leak-proof and clean container. To enhance the detection rate of AFB, bleach concentration technique was used according to the procedure explained in part two of Cheesbrough M [20]. Then the samples were stained using the Ziehl-Neelsen staining method for the diagnosis of AFB. TB positive sample was reported as positive if at least two AFB smear results were positive or one smear was positive with x-ray finding [19].

\subsection{Quality Control}

Internal quality control was done according to AFB smear microscopy manual [19]. Staining reagents were tested by known negative and positive control slides. All slides were read by experienced medical laboratory technologist, and the entire positive and 10\% of AFB negative slides were blindly re-checked by other qualified professionals.

\subsection{Ethical Approval and Consent to Participate}

Institutional ethical clearance was taken from research and ethics review board of Hawassa University. An official letter to obtain permission from the medical director of Hawassa Adare hospital was also written from the University before the data collection. The written consent and information about the purpose and importance of the study were given to each participant before the data collection. Privacy of the participants was secured at every point of the study. Patient participated in the research was on voluntary bases and those who were requested to quit their participation at any stage of the study were clued-up to do so without any limit.

\subsection{Data Processing and Analysis}

The gathered data were entered to Epi-Info version 3.5.1 and transferred to SPSS version 20.0 for additional analysis. Logistic regression analysis was used to assess the association between various variables and pulmonary tuberculosis. Odds ratios and 95\% CI were computed to determine the strength and presence of the association. 


\section{RESULTS}

\subsection{Socio-Demographic Characteristics of the Participants}

In the study, 207 diabetic patients participated with zero non-response rate. More than half of the participants were females 107 (51.7\%). The age of participants ranged from 17 to 95 years and the mean age was 48.7 years with \pm 11.5 standard deviation. The majority of participants were married $168(81.2 \%)$ and urban dwellers 180 (87.0\%). Regarding the educational status of participants, $66(31.9 \%)$ of them were illiterate and $49(23.7 \%)$ of them were educated up to the level of college and above. Nearly half of the participants 93 (44.6\%) were Orthodox religion followers followed by protestant religion which accounts about $34 \%$ of participants. Regarding the occupational status of the participant, 96 (46.4\%) of the participants were employed (government or private) in contrast, 87 (42\%) of them were unemployed. Monthly income of participants was also assessed and about $42 \%$ of the participants got less than 500 Ethiopian Birr per months and only about $10 \%$ of participants got more than 2000 Birr per month. More than two third of the study participants had 5 to 10 family member (Table 1).

Table 1. Socio- demographic characteristics of diabetic patient in Hawassa Adare Hospital, Southern Ethiopia, from March to May 2015 (n=207).

\begin{tabular}{|c|c|c|}
\hline Variable & Category & No. $(\%)$ \\
\hline \multirow[t]{2}{*}{ Sex } & Male & $100(48.3)$ \\
\hline & Female & $107(51.7)$ \\
\hline \multirow[t]{4}{*}{ Age in years [Mean: 48.7 years] } & $<35$ & $18(8.7)$ \\
\hline & $36-45$ & $74(35.7)$ \\
\hline & $46-55$ & $53(25.6)$ \\
\hline & $>55$ & $62(30.0)$ \\
\hline \multirow[t]{3}{*}{ Marital status } & Single & $29(14.0)$ \\
\hline & Married & $168(81.2)$ \\
\hline & Widowed & $10(4.8)$ \\
\hline \multirow[t]{4}{*}{ Religion } & Catholic & $8(3.8)$ \\
\hline & Muslim & $35(16.9)$ \\
\hline & Protestant & $71(34.3)$ \\
\hline & Orthodox & $93(44.9)$ \\
\hline \multirow[t]{4}{*}{ Educational status } & Not able to read & $66(31.9)$ \\
\hline & Primary school & $45(21.7)$ \\
\hline & Secondary school & $47(22.7)$ \\
\hline & College and above & $49(23.7)$ \\
\hline \multirow[t]{3}{*}{ No. of family } & $<5$ & $58(28.0)$ \\
\hline & $5-10$ & $142(68.6)$ \\
\hline & $>10$ & $7(3.4 \%)$ \\
\hline \multirow[t]{2}{*}{ Resident } & Urban & $180(87.0)$ \\
\hline & Rural & $27(13.0)$ \\
\hline \multirow[t]{4}{*}{ Monthly income [Ethio. Birr] } & $<500$ & $87(42.0)$ \\
\hline & $501-1000$ & $70(33.8)$ \\
\hline & $1001-2000$ & $28(13.5)$ \\
\hline & $>2000$ & $22(10.6)$ \\
\hline \multirow[t]{5}{*}{ Occupational status } & Employee & $96(46.4)$ \\
\hline & Student & $4(1.9)$ \\
\hline & Merchant & $7(3.4)$ \\
\hline & Farmer & $13(6.3)$ \\
\hline & Unemployed & $87(42.0)$ \\
\hline
\end{tabular}

\subsection{Medical Characteristic of DM Patients}

Of 207 participants, $171(82.61 \%)$ of them were with type two diabetes mellitus while the rest were type one. All patients had regular follow-up either treated by pills or insulin treatments. More than half 111 (53.6\%) of the participants were taking pills while The others were taking insulin as treatment. Duration of participants with DM after diagnosis ranged from 1 year to 27 years and the mean duration was 7.15 with 5 years standard deviation. Furthermore, 
the majority of the participants $84(40.6 \%)$ were with DM for less than five years after diagnosis while about $25 \%$ of them were living with the disease for more than 10 years. Of participants, $10(4.8 \%)$ of them were HIV positive. Regarding the body mass index of the participants, majority of $130(62.8 \%)$ were over-weight while only 17 participants were under-weight.

\subsection{Smear Positive Pulmonary TB in Diabetic Patients}

Out of 207 TB screened diabetic patients, 11 (5.3\%) [95\% CI: (2.2-8.4)] of them were smear-positive pulmonary TB by sputum concentration acid-fast stained direct microscopy technique. Of smear-positive patients, majority of them (72.7\%) were male patients and above fifty-five years old. Similarly, majority of them were married participants. A relatively highest proportion of smear-positive pulmonary tuberculosis was observed in age groups less than 35 years which accounts for about $15.78 \%$ (3/19) of the age group followed by age group older than 55 years with $6.4 \%(4 / 62)$ (Fig. 1). Proportion of smear positive pulmonary TB was almost similar in all educational statuses of participants but slightly high proportion was observed in participants who educated up to college and above. High proportion of pulmonary tuberculosis was exhibited in a participant who had more than ten family members per household than less family member. Proportion of tuberculosis was also determined in terms of income of study participants and high proportion was observed in patients with less than one thousand Ethiopian Birr per month. Relatively high proportion of smear positive pulmonary tuberculosis was detected among students followed by unemployed participants.

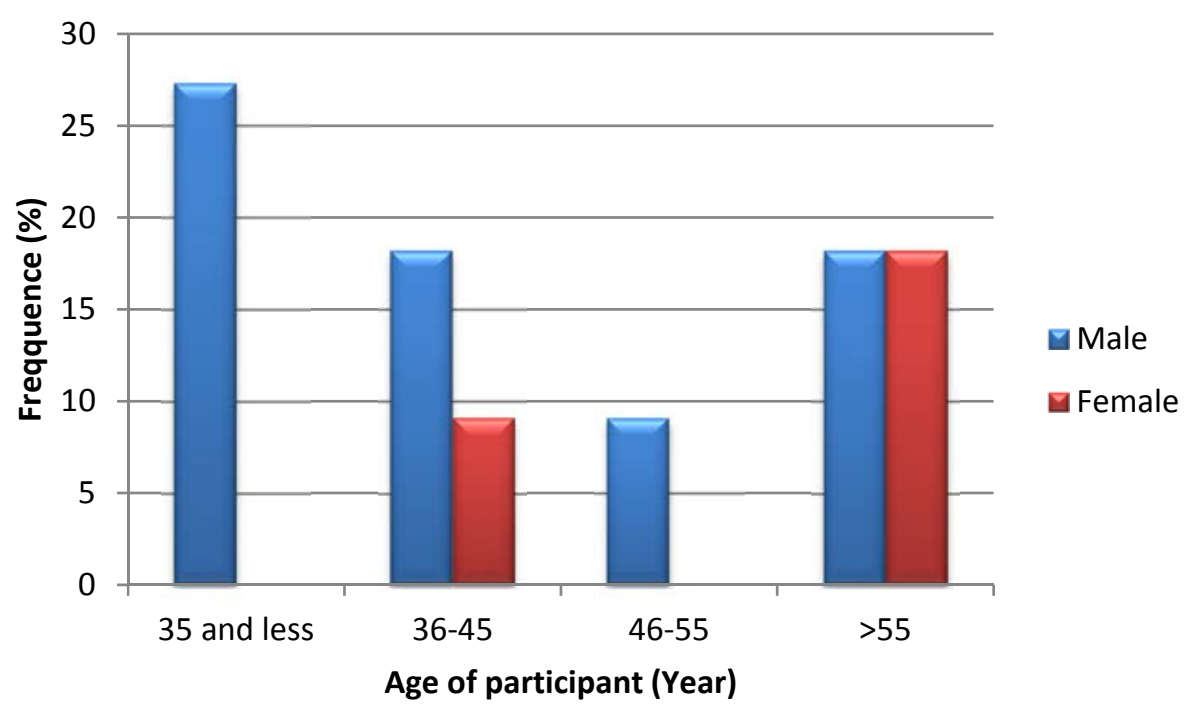

Fig. (1). Smear positive pulmonary tuberculosis by age and sex among diabetic patient in Hawassa Adare Hospital, Southern Ethiopia.

\subsection{Factor Associated with Smear-Positive Pulmonary Tuberculosis in Diabetic Patients}

In the bivariate analysis of logistic regression, statically significant association was observed between outcome variable and religion, duration of DM with patient, Body Mass Index (BMI), alcohol consumption habit, smoking habit, type of treatment for DM, contact history with TB, and HIV status of participants. In multivariable analysis, all sociodemographic characteristics of participants, smoking habit, and type of treatment for DM were insignificant. But, the duration of DM with patient, BMI, alcohol consumption habit, contact history with TB, and HIV status of participants were significantly associated with pulmonary tuberculosis among diabetic patients.

Diabetic patients who were underweight $[\mathrm{AOR}=9.94,95 \% \mathrm{CI}$ : $(1.51-80.89)]$ were about ten times more likely infected with pulmonary tuberculosis as compared to those who were overweight. Those patients living with DM for more than 10 years after DM diagnosed [AOR $=7.0395 \% \mathrm{CI}:(1.357,73.6)]$ were seven times more likely to have pulmonary tuberculosis as compared to those living with DM for less than five years. Alcohol consumption habit of diabetic patients revealed the odds of infection with pulmonary tuberculosis by about twelve times higher among alcohol drinkers than those who were non-drinkers $[\mathrm{AOR}=12.49,95 \% \mathrm{CI}:(3.28,77.94)]$. Diabetic patients who had contact history with tuberculosis $[\mathrm{AOR}=7.35,95 \% \mathrm{CI}$ : (1.1-39.12)] had about five times higher odds of infection with 
tuberculosis as compared to those patients without TB contact history. Being not infected with HIV among diabetic patients minimize the chance of being infected with pulmonary tuberculosis by about $93 \%$ [AOR $=0.074,95 \%$ CI: (0.001-0.29)] (Table 2).

Table 2. Factor associated with pulmonary tuberculosis among diabetic patient in Hawassa Adare Hospital, Southern Ethiopia, $2015(n=207)$.

\begin{tabular}{|c|c|c|c|c|c|}
\hline \multirow{2}{*}{ Variables } & \multicolumn{2}{|c|}{ TB status } & \multicolumn{2}{|c|}{ Odds ratio $(95 \% \mathrm{CI})$} & \multirow{2}{*}{ P-Value } \\
\hline & Smear Positive & Smear Negative & Crude & Adjusted & \\
\hline \multicolumn{6}{|c|}{ Duration with DM (In year) } \\
\hline$<5$ & 2 & 82 & Ref. & Ref. & \\
\hline 6-10 & 3 & 67 & $1.836(0.29,11.31)$ & $11.62(0.89,51.63)$ & 0.061 \\
\hline$>10$ & 6 & 47 & $5.23(1.02,26.98)$ & $7.03(1.357,73.6)$ & 0.028 \\
\hline \multicolumn{6}{|c|}{ Body mass index $\left(\mathrm{Kg} / \mathrm{m}^{2}\right)$} \\
\hline$<18$ & 3 & 14 & $6.75(1.37,33.29)$ & $9.94(1.51,80.89)$ & 0.024 \\
\hline $18-24.5$ & 4 & 56 & $2.25(0.54,9.32)$ & $7.09(0.54,53.16)$ & 0.136 \\
\hline$>24.5$ & 4 & 126 & Ref. & Ref. & \\
\hline \multicolumn{6}{|c|}{ Alcohol consumption habit } \\
\hline Yes & 8 & 23 & $10.58(2.96,41.05)$ & $12.49(3.28,77.94)$ & 0.002 \\
\hline No & 3 & 173 & Ref. & Ref. & \\
\hline \multicolumn{6}{|c|}{ Smoking habit* } \\
\hline Yes & 7 & 11 & $29.47(7.47,115.91)$ & $4.81(0.29,59.04)$ & \\
\hline No & 4 & 185 & \begin{tabular}{c|} 
Ref. \\
\end{tabular} & Ref. & \\
\hline \multicolumn{6}{|c|}{ Type of treatment* } \\
\hline Pills & 3 & 108 & $0.31(0.079,1.19)$ & $0.29(0.039,2.23)$ & \\
\hline Insulin & 8 & 88 & Ref. & Ref. & \\
\hline \multicolumn{6}{|c|}{ Contact history with TB } \\
\hline Yes & 7 & 19 & $7.303(3.37,40.81)$ & $5.35(1.1,39.12)$ & 0.04 \\
\hline No & 4 & 177 & Ref. & Ref. & \\
\hline \multicolumn{6}{|l|}{ HIV status } \\
\hline Negative & 7 & 190 & $0.055(0.013,0.241)$ & $0.074(0.001,0.29)$ & 0.006 \\
\hline Positive & 4 & 6 & Ref. & Ref. & \\
\hline
\end{tabular}

*=Not significant in backward stepwise logistic regression; Ref.: Reference

\section{DISCUSSION}

The overall prevalence of pulmonary TB in diabetics was shown to be $5.3 \%$ in our study which was higher than the estimated prevalence of TB in total population [1]. This finding is in line with the studies conducted in Tanzania (5.4\%) [22] and India (6\%) [23]. Moreover, our finding is also comparable to the study carried out in Dessie, northeast part of Ethiopia (6.2\%) [18] and a study conducted in Addis Ababa (5.8\%) [24]. However, it is lower than the findings from Pakistan (14\%) [25] and South Africa (10.6\%) [13]. In contrast, our finding is higher than the finding of a study conducted in India (2.6\%) [26]. The observed difference in the pulmonary tuberculosis prevalence among diabetic patients in Pakistan might be due to the difference in the severity of diabetic patient which were admitted in the study while in South Africa, they also use the radiographic diagnostic method. Moreover, the difference with the low prevalence of tuberculosis in Indian diabetic patients might be due to the difference in socio-demographic characteristic of the population as well as due to the difference in detection ability of the diagnostic methods.

Patients living with DM are at high risk of developing active tuberculosis which may impair both innate and adaptive immune responses to Mycobacterium tuberculosis. The relationship between neutrophils and autoimmune diabetes has also been documented. The number of macrophages is low in diabetes as compared non-diabetes and macrophages are important for the activation of the cellular immune response [6]. The increased risk of TB associated with diabetes may be also attributed to the chronic nature of the illness and failure to control glucose that may enhance the risk of TB $[5,10,21]$.

In the current study, duration of patient with DM was associated with the occurrence of pulmonary tuberculosis infection in diabetic patients. Those diabetic patients live with DM for more than ten years after they had been 
diagnosed for DM had seven times high odds of infection with TB than those patient living with DM for less than five years $[\mathrm{AOR}=7.0395 \% \mathrm{CI}:(1.357,73.6)]$. This finding is similar to a study conducted in Dessie, Ethiopia [AOR: 8.89 ; 95\% CI: (1.88-58.12)] [18] and a study conducted in Pakistan [14]. This may be due to the complication of DM when in long duration. The immunity of the patient is also more compromised ina patient living with DM for a long time as a result of microvascular disease and macrovascular disease as well as treatment tolerance of the disease.

The other finding of the current study revealed that those DM patients who were underweight $\left(\mathrm{BMI}<18.5 \mathrm{Kg} / \mathrm{m}^{2}\right)$ had about ten times high odds infection with DM as compared to those with body weight gains [9.94, 95\% CI: (1.51, 80.89)]. Similar finding was obtained in a study conducted in Chiayi, Taiwan [OR $=6.635,95 \%$ CI: (2.096-21.007)] [15], a study conducted in India [AOR $=2.03,95 \%$ CI: $(1.32-3.12)$ ] [16] and in Tanzania [OR = 2.08 955 CI: (1.064.06)] [27]. BMI indicates malnutrition which is a factor for a number of infectious diseases. Underweight individuals have a weak immunity system which expose them to infection.

In the present study, there was a statically significant relation between alcohol consumption habit and pulmonary tuberculosis in diabetics. A Study conducted in Taiwan also showed that consumption was an associated factor for tuberculosis infection in male diabetics $[\mathrm{OR}=2.410,95 \% \mathrm{C}:(1.425-4.074)]$ [15]. The other study conducted in India also showed an association between alcohol consumption and TB infection [28]. A systematic review also indicated the association between alcohol addiction and tuberculosis [29]. A number of reasons have been documented concerning their association. One of these reasons is the toxic effects of alcohol on the immune system making the host more susceptible to TB infection. In animal studies, the chronic and acute alcohol consumption directly impaired cellmediated immunity and macrophage functions [29].

Contact history of diabetic patients with TB had a significant association with pulmonary tuberculosis; those diabetic patients who responded to their contact with TB infected person had about five times high odds of infection with TB $[$ AOR $=5.35, \mathrm{CI}:(1.1,39.12)]$. Similar finding was observed in a study conducted in Dessie, Ethiopia $[(\mathrm{AOR}=9.4 ; 95 \% \mathrm{CI}:(1.822-48.50)]$ [18]. Systematic review also showed that contacts with tuberculosis patients are a high-risk group for developing TB [30]. Even though some studies showed the prevalence of latent TB infection and TB among contacts is significantly less in developed countries, diabetic patient's contact with TB has a significant association in our study. This might be due to the impaired immune system in diabetic patients that makes them susceptible to various infections [30]. Being HIV positive was also associated with pulmonary TB infection in diabetics in this study. Similar finding was observed in a study conducted in rural south Indian [31] and in Ethiopia [32]. Another study conducted in India also showed a strong association among TB, diabetics and HIV [33].

Social desirability bias and recall bias are the potential limitations of this study. The lack of sufficient study in Ethiopian context made the comparison of the results difficult. Even though direct microscopic examination is not a gold standard method, it is highly utilized in the diagnosis of pulmonary tuberculosis in developing countries. It is a less sensitive method and it may underestimate the pulmonary TB prevalence unless it is supported by sputum concentration method. In our study to modify its sensitivity, we conducted the direct AFB stain with Sputum concentration technique.

\section{CONCLUSION}

Prevalence of pulmonary tuberculosis in the diabetic patient was high as compared to the national estimated prevalence of TB in the total population. Duration of patients with DM after they have been diagnosed, being underweight, alcohol consumption habit, and contact history with tuberculosis were positively associated with pulmonary TB infection while being HIV seronegative, they had been negatively associated with pulmonary tuberculosis infection in diabetic patients. By considering these findings it is recommended that TB control programs, policy makers and stakeholders should increase their efforts.

\section{LIST OF ABBREVIATIONS}

$\begin{array}{lll}\text { AFB } & = & \text { Acid Fast Bacilli } \\ \text { AOR } & = & \text { Adjusted Odd Ratio } \\ \text { BMI } & = & \text { Body Mass Index } \\ \text { CI } & = & \text { Confidence Interval } \\ \text { DM } & = & \text { Diabetic Mellitus } \\ \text { HIV } & = & \text { Human Immunodeficiency Virus } \\ \text { SPPTB } & = & \text { Smear Positive Pulmonary Tuberculosis }\end{array}$


TB

WHO
Tuberculosis

$=\quad$ World Health Organization

\section{AUTHORS' CONTRIBUTIONS}

Ademe Abera: Conducted data collection, participated in the design of the study, revised subsequent drafts of the paper. Gemechu Ameya: Participated in the design of the study, analyzed the data and drafted the manuscript. Both authors have read and approved the final manuscript.

\section{ETHICS APPROVAL AND CONSENT TO PARTICIPATE}

Institutional ethical clearance was taken from research and ethics review board of Hawassa University. An official letter to obtain permission from the medical director of Hawassa Adare hospital was also written from the University before the data collection.

\section{HUMAN AND ANIMAL RIGHTS}

Animals did not participate in this research. All human research procedures followed were in accordance with the ethical standards of the committee responsible for human experimentation (institutional and national), and with the Helsinki Declaration of 1975, as revised in 2008.

\section{CONSENT FOR PUBLICATION}

The written consent and information about the purpose and importance of the study were given to each participant before the data collection.

\section{CONFLICT OF INTEREST}

The author declares no conflict of interest, financial or otherwise.

\section{ACKNOWLEDGMENTS}

We are grateful to Hawassa Adare hospital for permission of data collection and the staff of Hawassa Adare hospital laboratory staffs for their assistance during data collection.

\section{REFERENCES}

[1] World Health Organization. Global tuberculosis report 2015. WHO/HTM/TB/2015.22 Geneva, Switzerland: WHO, 2015. Available from: http:// apps.who.int/ iris/bitstream/ 10665/191102/1/

[2] Stevenson CR, Forouhi NG, Roglic G, et al. Diabetes and tuberculosis: The impact of the diabetes epidemic on tuberculosis incidence. BMC Public Health 2007; 7: 234. [http://dx.doi.org/10.1186/1471-2458-7-234] [PMID: 17822539]

[3] World Health Organization. Global report on diabetes. Geneva, Switzerland: WHO, 2016. Available from: http:// www.who.int/ diabetes/ global-report/ WHD16-press-release-EN_3.pdf?ua=1

[4] International Diabetic Federation. Diabetes in Ethiopia-2015. Available from: http:// www.idf.org/ membership/ afr/ethiopia Accessed August 31,2016

[5] Baghaei P, Marjani M, Javanmard P, Tabarsi P, Masjedi MR. Diabetes mellitus and tuberculosis facts and controversies. J Diabetes Metab Disord 2013; 12(1): 58 [http://dx.doi.org/10.1186/2251-6581-12-58] [PMID: 24360398]

[6] García-Elorriaga G, Del Rey-Pineda G. Type 2 diabetes mellitus as a risk factor for tuberculosis. J Mycobac Dis 2014 ; 4 : 144.

[7] Jeon CY, Murray MB. Diabetes mellitus increases the risk of active tuberculosis: A systematic review of 13 observational studies. PLoS Med 2008; 5(7): e152.

[http://dx.doi.org/10.1371/journal.pmed.0050152] [PMID: 18630984]

[8] Pealing L, Wing K, Mathur R, Prieto-Merino D, Smeeth L, Moore DAJ. Risk of tuberculosis in patients with diabetes: Population based cohort study using the UK Clinical Practice Research Datalink. BMC Med 2015; 13: 135. [http://dx.doi.org/10.1186/s12916-015-0381-9] [PMID: 26048371]

[9] World Health Organization. Global strategy and targets for tuberculosis prevention, care and control after 2015. The End TB Strategy. WHO, 2015. Available from: http://www.who.int/ tb/strategy/End_TB_Strategy.pdf?ua=1

[10] Mboussa J, Monabeka H, Kombo M, Yokolo D, Yoka-Mbio A, Yala F. Course of pulmonary tuberculosis in diabetics. Rev Pneumol Clin 2003; 59(1): 39-44.

[PMID: 12717325] 
[11] Dooley KE, Chaisson RE. Tuberculosis and diabetes mellitus: Convergence of two epidemics. Lancet Infect Dis 2009; 9(12): 737-46. [http://dx.doi.org/10.1016/S1473-3099(09)70282-8] [PMID: 19926034]

[12] Buli GA, Lukas FY, Amenta NW. Magnitude of Tuberculosis Lymphadenitis in Hawassa University referral hospital, Southern Ethiopia. Am J Pharm Health Res 2015; 3(2): 116-20. [http://dx.doi.org/10.11648/j.ajhr.20150302.19]

[13] Dobler CC, Flack JR, Marks GB. Risk of tuberculosis among people with diabetes mellitus: An Australian nationwide cohort study. BMJ Open 2012; 2(1): e000666. [http://dx.doi.org/10.1136/bmjopen-2011-000666] [PMID: 22331390]

[14] Jabbar A, Hussain SF, Khan AA. Clinical characteristics of pulmonary tuberculosis in adult Pakistani patients with co-existing diabetes mellitus. East Mediterr Health J 2006; 12(5): 522-7. [PMID: 17333789]

[15] Lin YH, Chen CP, Chen PY, et al. Screening for pulmonary tuberculosis in type 2 diabetes elderly: A cross-sectional study in a community hospital. BMC Public Health 2015; 15: 3. [http://dx.doi.org/10.1186/1471-2458-15-3] [PMID: 25572102]

[16] Viswanathan V, Kumpatla S, Aravindalochanan V, et al. Prevalence of diabetes and pre-diabetes and associated risk factors among tuberculosis patients in India. PLoS One 2012; 7(7): e41367. [http://dx.doi.org/10.1371/journal.pone.0041367] [PMID: 22848473]

[17] Federal Democratic Republic of Ethiopia, Population census commission and UNFPA Summary and Statistical report of the 2007 Population and Housing Census. Addis Ababa, Ethiopia. 2008

[18] Amare H, Gelaw A, Anagaw B, Gelaw B. Smear positive pulmonary tuberculosis among diabetic patients at the Dessie referral hospital, Northeast Ethiopia. Infect Dis Poverty 2013; 2(1): 6. [http://dx.doi.org/10.1186/2049-9957-2-6] [PMID: 24499664]

[19] Ministry of Health. AFB smears microscopy manual. Ethiopian health and nutrition research institute Addis Ababa, Ethiopia.2009; 4th: pp.10-45.

[20] Cheesbrough M. District laboratory practice in tropical countries Part 2. $2^{\text {nd }}$ ed. New York Cambridge University Press 2006; pp. 71-6. [http://dx.doi.org/10.1017/CBO9780511543470]

[21] Workneh MH, Bjune GA, Yimer SA. Prevalence and associated factors of diabetes mellitus among tuberculosis patients in south-eastern amhara region, ethiopia: A cross sectional study. PLoS One 2016; 11(1): e0147621. [http://dx.doi.org/10.1371/journal.pone.0147621] [PMID: 26808967]

[22] Rao P. Persons with type 2 diabetes and co-morbid active tuberculosis should be treated with insulin in Tanzania. Int J Diabetes Dev Ctries 1999; $19: 3-5$

[23] Lalit K. Diabetes mellitus and tuberculosis the brewing double trouble. Indian Journal Tuberculosis New Delhi 2003; 50(Suppl. 4): 1-2.

[24] Feleke Y, Abdulkadir J, Aderaye G. Prevalence and clinical features of tuberculosis in Ethiopian diabetic patients. East Afr Med J 1999; 76(7): 361-4. [PMID: 10520361]

[25] Amin S, Khattak MI, Shabbier G, Wazir MN. Frequency of pulmonary tuberculosis in patients with diabetes mellitus. Gomal Journal of Medical Sciences 2011; 9(2): 163-5.

[26] Kumar A, Gupta D, Burugina S, Nair A, Satyanarayana S, Kumar AM, et al. Screening of patients with diabetes mellitus for tuberculosis in India. Trop Med Int Health 2013; 18(5): 646-54.

[27] Faurholt-Jepsen D, Range N, PrayGod G, et al. The role of anthropometric and other predictors for diabetes among urban Tanzanians with tuberculosis. Int J Tuberc Lung Dis 2012; 16(12): 1680-5. [http://dx.doi.org/10.5588/ijtld.12.0360] [PMID: 23131269]

[28] Suhadev M, Thomas BE, Raja Sakthivel M, et al. Alcohol use disorders (AUD) among tuberculosis patients: A study from Chennai, South India. PLoS One 2011; 6(5): e19485.

[http://dx.doi.org/10.1371/journal.pone.0019485] [PMID: 21611189]

[29] Lönnroth K, Williams BG, Stadlin S, Jaramillo E, Dye C. Alcohol use as a risk factor for tuberculosis - A systematic review. BMC Public Health 2008; 8: 289.

[http://dx.doi.org/10.1186/1471-2458-8-289] [PMID: 18702821]

[30] Fox GJ, Barry SE, Britton WJ, Marks GB. Contact investigation for tuberculosis: A systematic review and meta-analysis. Eur Respir J 2013; 41(1): 140-56 [http://dx.doi.org/10.1183/09031936.00070812] [PMID: 22936710]

[31] Gupta S, Shenoy VP, Bairy I, Srinivasa H, Mukhopadhyay C. Diabetes mellitus and HIV as co-morbidities in tuberculosis patients of rural south India. J Infect Public Health 2011; 4(3): 140-4. [http://dx.doi.org/10.1016/j.jiph.2011.03.005] [PMID: 21843860]

[32] Assefa G, Solomon M, Shiatye A, Hanan Y. High magnitude of diabetes mellitus among Active Pulmonary Tuberculosis Patients in Ethiopia. Br J Med Med Res 2013; 4(3): 862-72. 
[33] Sekar R, Mythreyee M. Tuberculosis, HIV/AIDS and diabetes - is it time to think together? J Medical Microbiol Diagnosis 2012 ; 1: e103. [http://dx.doi.org/10.4172/2161-0703.1000e103]

(C) 2018 Abera and Ameya.

This is an open access article distributed under the terms of the Creative Commons Attribution 4.0 International Public License (CC-BY 4.0), a copy of which is available at: (https://creativecommons.org/licenses/by/4.0/legalcode). This license permits unrestricted use, distribution, and reproduction in any medium, provided the original author and source are credited. 